\title{
Orchidopexy for Testicular Torsion: A Systematic Review of
}

\section{Surgical Technique}

Moore $\mathrm{SL}^{1,2}$, Chebbout $\mathrm{R}^{1}$, Cumberbatch $\mathrm{M}^{1,3}$, Bondad $\mathrm{J}^{1,4}$, Forster $\mathrm{L}^{1,5}$, Hendry $\mathrm{J}^{1,6}$, Lamb $B^{1,7}$, MacLennan $S^{1,8}$, Nambiar $A^{1,9}$, Shah $\mathrm{TT}^{1,10}$, Stavrinides $\mathrm{V}^{1,11}$, Thurtle $\mathrm{D}^{1,12}$, Pearce $\mathrm{I}^{1,13}$, Kasivisvanathan $\mathrm{V}^{1,14,15}$

1. British Urology Researchers in Surgical Training (BURST) Research Collaborative

2. North Wales Clinical Research Centre/Wrexham Maelor Hospital, Betsi Cadwaladr University Health Board, Wales, UK.

3. Department of Urology, Sheffield Teaching Hospitals NHS Foundation Trust, UK.

4. Department of Urology, Lister Hospital, Stevenage, UK.

5. Department of Urology, Royal Free Hospital, London, UK.

6. Department of Urology, Queen Elizabeth University Hospital, Glasgow, Scotland, UK.

7. Department of Urology, Cambridge University Hospitals NHS Foundation Trust, UK.

8. Academic Urology Unit, University of Aberdeen, Scotland, UK.

9. Department of Urology, Freeman Hospital, Newcastle-upon-Tyne, UK.

10. Charing Cross Hospital, Imperial College Healthcare NHS Trust and Imperial Prostate, Department of Surgery and Cancer, Imperial College London, UK.

11. Division of Surgery and Interventional Science, University College London, UK.

12. Academic Urology Group, Department of Surgery, University of Cambridge, Cambridge, UK.

13. Manchester Royal Infirmary, Manchester University NHS Foundation Trust, UK.

14. Division of Surgery and Interventional Science, University College London, UK.

15. Department of Urology, University College London Hospital, London, UK

Key words: Testicular torsion; Orchidopexy; Surgical technique; Systematic review

Word count: 2936 words

Corresponding Author: Mr Veeru Kasivisvanathan (veeru.kasi@ucl.ac.uk)

Conflicts of interest: None.

Funding: None to declare. 


\section{Abstract}

\section{Context}

Acute testicular torsion is a common urological emergency. Accepted practice is surgical exploration, detorsion and orchidopexy for a salvageable testis.

\section{Objective}

To critically evaluate methods of orchidopexy and their outcomes with a view to determining optimal surgical technique.

\section{Evidence Acquisition}

The review protocol was published via PROSPERO [CRD42016043165] and conducted in accordance with PRISMA. EMBASE, MEDLINE and CENTRAL databases were searched using terms: 'orchidopexy', 'fixation', 'exploration', 'torsion', 'scrotum' and variants. Article screening was performed by two reviewers independently. The primary outcome was retorsion rate of the ipsilateral testis following orchidopexy. Secondary outcomes included testicular atrophy and fertility.

\section{Evidence Synthesis}

To our knowledge, this is the first systematic review on this topic. The search yielded 2257 abstracts. Five studies ( $n=138$ patients) were included.

All five techniques differed in incision and/or type of suture and/or point(s) of fixation. Postoperative complications were reported in one study and included scrotal abscess in $9.1 \%$ and stitch abscess in $4.5 \%$. The contralateral testis was fixed in $57.6 \%$ of cases.

Three studies reported follow-up duration (range 6-31 weeks). No study reported any episodes of ipsilateral retorsion. In the studies reporting ipsilateral atrophy rate, this ranged from 9.147.5\%. Fertility outcomes and patient reported outcome measures were not reported in any studies. 


\section{Conclusion}

There is limited evidence in favour of any one surgical technique for acute testicular torsion. During the consent process for scrotal exploration, the uncertainties in the long-term harms should be discussed. This review highlights the need for an interim consensus in surgical approach until robust studies examining the effects of operative approach on clinical and fertility outcomes are available.

\section{Patient Summary}

We reviewed the literature to assess outcomes of using various surgical techniques to fix the twisting of the testis. Our review shows that there is limited evidence in favour of any one technique.

Abstract word count: 299 


\section{Introduction}

Testicular torsion is a common urological and paediatric emergency, and results in ischaemia due to twisting of the spermatic cord and thus interruption of blood flow [1]. It accounts for around $17-35 \%$ of patients presenting with an acute scrotum and affects up to 4.5 in every 100,000 men below the age of 25 , each year, with peaks of incidence during the perinatal and pubertal ages [2-5]. The anatomical configuration of a "Bell Clapper" deformity is commonly cited as a predisposing factor and other proposed mechanisms include physical activity [e.g. cycling], trauma, or genetic inheritance [2,6-8]. If untreated, testicular torsion will result in permanent ischaemic injury, $[9,10]$, thus suspected testicular torsion requires immediate surgical exploration [3].

On surgical exploration, testicular torsion can usually be subdivided into intravaginal and extravaginal subtypes [1]. Time to presentation is crucially important, with pain lasting $>4-8$ hours being highly associated with testicular death without intervention [2]. In approximately one in three cases, the testis is considered dead resulting in orchidectomy (orchiectomy) $[11,12]$. Following surgical exploration and detorsion, a salvageable testis will undergo orchidopexy (orchiopexy), and usually the contralateral testis will also undergo orchidopexy [13].

The literature on orchidopexy technique is heterogenous and scarce, with variation reported in type of suture use, fixation methods, synchronous procedures and contralateral testicular fixation. Recurrent ipsilateral or contralateral torsion, testicular ischaemia and atrophy and negative impacts on fertility have all been reported as possible complications after orchidopexy $[14,15]$. Despite these complications, the significant potential for avoidable harm, and possible

medicolegal implications resulting from sub-optimal management, there is a paucity in published synthesis of evidence comparing the different surgical techniques [16]. 
The aim of this study was to describe, summarise and evaluate the published data reporting surgical technique and subsequent outcomes of orchidopexy indicated for acute testicular torsion with a view to determining optimal surgical technique.

\section{Evidence Acquisition}

\section{Protocol and registration}

The protocol of the study was decided a priori and published on the online systematic review register PROSPERO (registration number: CRD42016043165).

\section{Eligibility criteria}

All studies reporting orchidopexy indicated for acute testicular torsion in an adult and/or paediatric population were considered for inclusion. In order to be eligible for inclusion, studies were required to report the detailed surgical technique used to perform the orchidopexy along with data pertaining to at least one of the stated outcome measures specified below. Surgical technique was deemed to include: approach, suture type, number of points of fixation, anatomic location of fixation points and use of Jaboulay pouch or vaginalis patch technique. The Jaboulay pouch technique refers to the excision and eversion of the tunica vaginalis. The vaginalis patch technique refers to the use of a tunica vaginalis patch graft during the procedure. Included studies were permitted to be interventional or observational and retrospective or prospective. An interventional study refers to any study that involves exposing study participants to a specific treatment with the outcome measured. An observational study refers to any study describing outcomes from current/routine practice, whether that be in a prospective or retrospective manner

Studies reporting orchidopexy indicated for anything other than acute testicular torsion, for example cryptochordism or intermittent testicular torsion, were excluded. Any data pertaining to orchidopexy in neonates (age less than 30 days) was not included. When data could not be used to answer at least one of the stated primary or secondary outcomes, the study was not 
included. Case reports, reviews, comments, editorials, abstracts or conference proceedings only were excluded.

\section{Outcomes}

The primary outcome was torsion recurrence rate of the ipsilateral testicle following orchidopexy for acute testicular torsion. Acute testicular torsion was defined as presentation of an acute scrotum with confirmed testicular torsion found intra-operatively. Torsion recurrence was defined as a re-presentation of acute testicular torsion of the previously fixated testicle.

Secondary outcomes included testicular atrophy of ipsilateral testicle, ipsilateral torsion recurrence rate, contralateral torsion, testicular atrophy of the contralateral testicle following orchidopexy and fertility outcomes following orchidopexy for testicular torsion. Testicular atrophy was defined by new reduction in size in comparison to contralateral testicle found on clinical examination and/or Doppler ultrasound. Fertility was inferred from changes in serum hormone markers and/or semen analysis/spermiogram [15, 26, 27].

\section{Search Strategy}

The EMBASE, MEDLINE and CENTRAL literature bases were searched using the following search terms, combined using Boolean operators as appropriate: 'orchiopexy', 'orchidopexy', 'fixation', 'exploration', 'spermatic cord torsion', 'torsion', 'scrotum', 'acute scrotum'. There were no limits applied to the search, and the search was duplicated by two authors independently to ensure identical results.

\section{Study selection}


A team of six authors independently screened the abstracts and full papers using the online systematic review platform Covidence (www.covidence.org). To ensure consistency between reviewers, a pilot of a random selection of the abstracts and full papers was first screened by all authors before initiation of the real screening process. Any conflicts in decisions were resolved by author M.C. Reference lists of key studies in the field were hand-searched for other relevant studies.

\section{Data extraction and synthesis}

A data collection proforma was designed with input from all authors a priori with desired data fields based on study outcomes and predicted sources of heterogeneity and confounding variables. Data was extracted independently by two authors, with any conflicts in data extraction resolved by author M.C.

Data were collected for the following fields: study design, demographic data, intraoperative details, surgical technique, follow-up, and outcome results. Study design data included methodology, year of publication, number of patients, and number of orchidopexies. Demographic data included previous testicular surgery and surgeon specialty and experience. Intra-operative data included anaesthetic type, testicle colour, mean degree of torsion, active bleeding and operation duration. The exact wording of the surgical technique of each article was recorded, as well as specific information on operative approach, suture material, suture size, number of sutures, points of fixation, removal of testicular appendage, use of the Jaboulay procedure or vaginal patch technique, and contralateral testicle fixation. Follow-up type, frequency and duration were recorded, and data pertaining to the primary and secondary outcomes was detailed.

\section{Risk of bias}


A full risk of bias assessment was undertaken using the ROBINS-1 tool as per the Cochrane collaboration guidelines.

\section{Evidence Synthesis}

\section{Demographics}

The search was conducted in accordance with PRISMA guidelines and yielded 2257 results, with seven studies suitable for inclusion (figure 1) [17-23]. Publication year ranged from 19752012, with three UK-based studies, two US-based studies, one Jamaican study and one German study. Four studies were from urology department settings, two from paediatric surgery settings, and one from a general surgery setting. Six of the seven studies were retrospective. One study reviewed notes dating back over a fourteen year period, three studies reviewed notes dating back over a ten-year period, whilst one study reviewed back over a sixyear period, one over a two-year period and one over a one-year period.

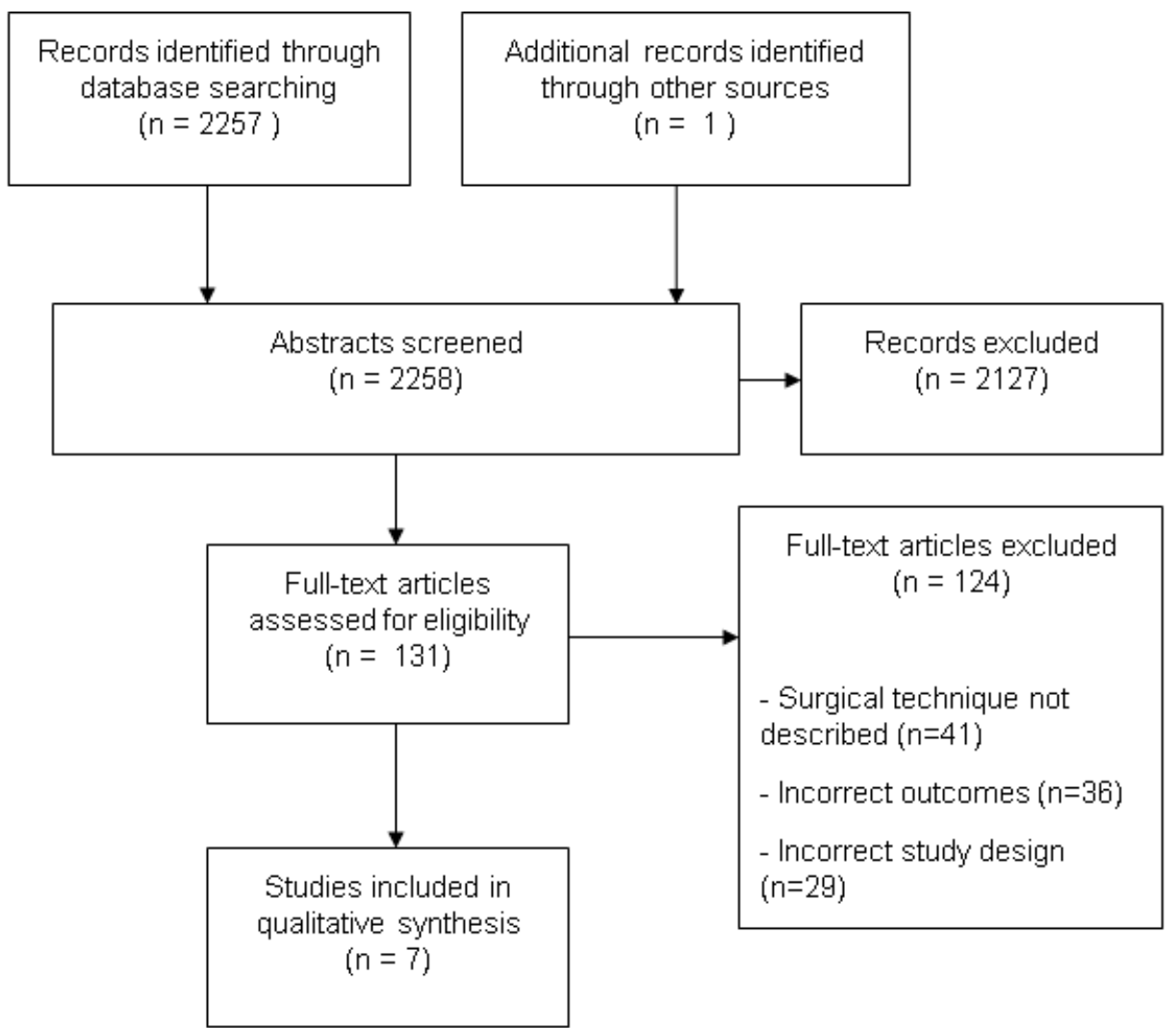


Figure 1 - PRISMA diagram

A total of 182 patients were included in this review (range 10-65 patients). Two studies reported mean age that can be attributed to the included cohort only (10.1 years and 14.0 years) $[19,21]$. The total number of orchidopexies was 266 (range 19-92 procedures). Onehundred-and-five out of one-hundred-and-eighty-two (105/182 (57.7\%)) patients underwent contemporaneous bilateral orchidopexy.

Risk of bias assessment using ROBINS-I highlighted a high risk of bias in all studies (figures 2 and 3).
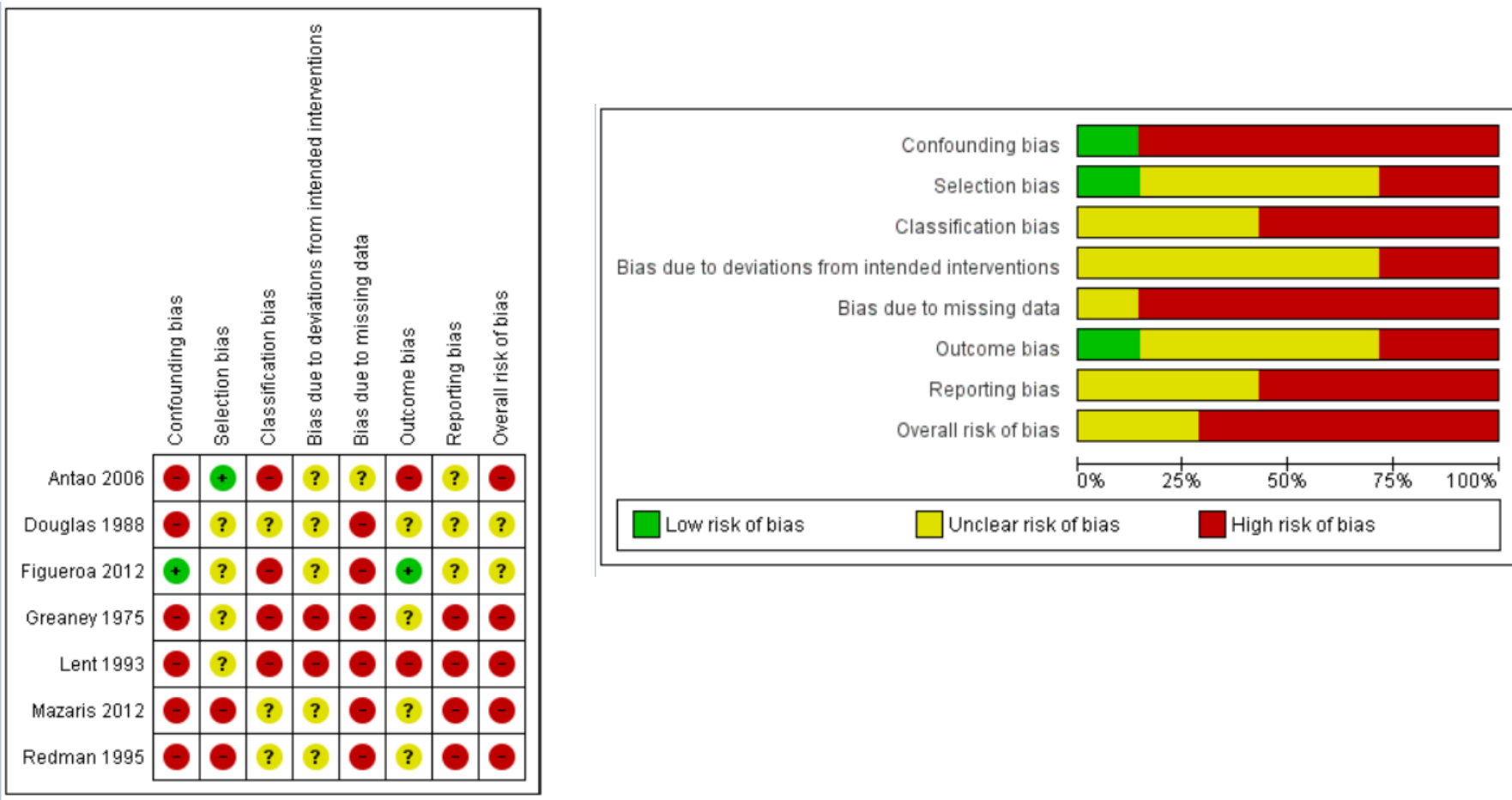

Figures 2 and 3 - risk of bias assessments undertaken using the ROBINS- 1 criteria.

\section{Operative technique}

The exact procedure for each method of orchidopexy is described in table 1 . Three studies reported using non-absorbable sutures [17,18,21], whilst one reported using absorbable sutures [20], one reported using both [23], one reported eversion and suturing of the tunica 
vaginalis alone [19], and one did not report suture material [22]. Removal of testicular appendage was not reported in any of the studies. Jaboulay technique was reported to have been undertaken in three studies $[19,20,23]$, while one study reported vaginalis patch [22]. Figures 4-10 demonstrate some of the key differences between techniques described (where enough detail on operative technique was provided to allow for accurate reproduction of diagrams). 


\begin{tabular}{|c|c|c|c|c|c|c|c|c|c|}
\hline Study & Design & Surgical technique & $\mathbf{N}$ & Age & $\begin{array}{l}\text { of } \\
\text { torsion }\end{array}$ & Complications & $\begin{array}{l}\text { of follow-up } \\
\text { [weeks] }\end{array}$ & $\begin{array}{l}\text { retorsion } \\
\text { rate [\%] }\end{array}$ & $\begin{array}{l}\text { testicular } \\
\text { atrophy }\end{array}$ \\
\hline $\begin{array}{l}\text { Greaney } \\
1975 \\
{[17]}\end{array}$ & $\begin{array}{l}\text { Retrospective } \\
\text { case series }\end{array}$ & $\begin{array}{l}\text { Incision: NR. } \\
\text { Fixation: Interrupted thread sutures to } \\
\text { maintain upright position. } \\
\text { Closure: Eversion of tunica vaginalis, } \\
\text { scrotum closed with catgut to the dartos and } \\
\text { clips to skin. }\end{array}$ & 22 & NR & NR & $\begin{array}{l}\text { Local } \\
\text { inflammation } \\
(3 / 22,13.6 \%) \\
\text { Scrotal abscess } \\
\text { formation }(2 / 22 \text {, } \\
9.1 \%) \\
\text { Stich abscess } \\
(1 / 22,4.5 \%)\end{array}$ & NR & 0 & 2 \\
\hline $\begin{array}{l}\text { Douglas } \\
1988 \\
{[18]}\end{array}$ & $\begin{array}{l}\text { Retrospective } \\
\text { case series }\end{array}$ & $\begin{array}{l}\text { Incision: Transverse incision to scrotum and } \\
\text { tunica vaginalis. } \\
\underline{\text { Fixation: } 4-0 \text { non-absorbable suture. }} \\
\underline{\text { Closure: Tunica albuginea incorporated into }} \\
\text { tunica vaginalis during closure. }\end{array}$ & 36 & NR & NR & 0 & NR & 0 & NR \\
\hline $\begin{array}{l}\text { Lent } \\
1993 \\
{[19]}\end{array}$ & $\begin{array}{l}\text { Retrospective } \\
\text { case series }\end{array}$ & $\begin{array}{l}\text { Incision: Transverse incision to scrotum } \\
\text { and tunica vaginalis. } \\
\text { Fixation: A continuous suture to the margins } \\
\text { of the tunica vaginalis communis without } \\
\text { additional fixation to the testis. }\end{array}$ & $32^{*}$ & NR & NR & NR & NR & 0 & 0 \\
\hline
\end{tabular}




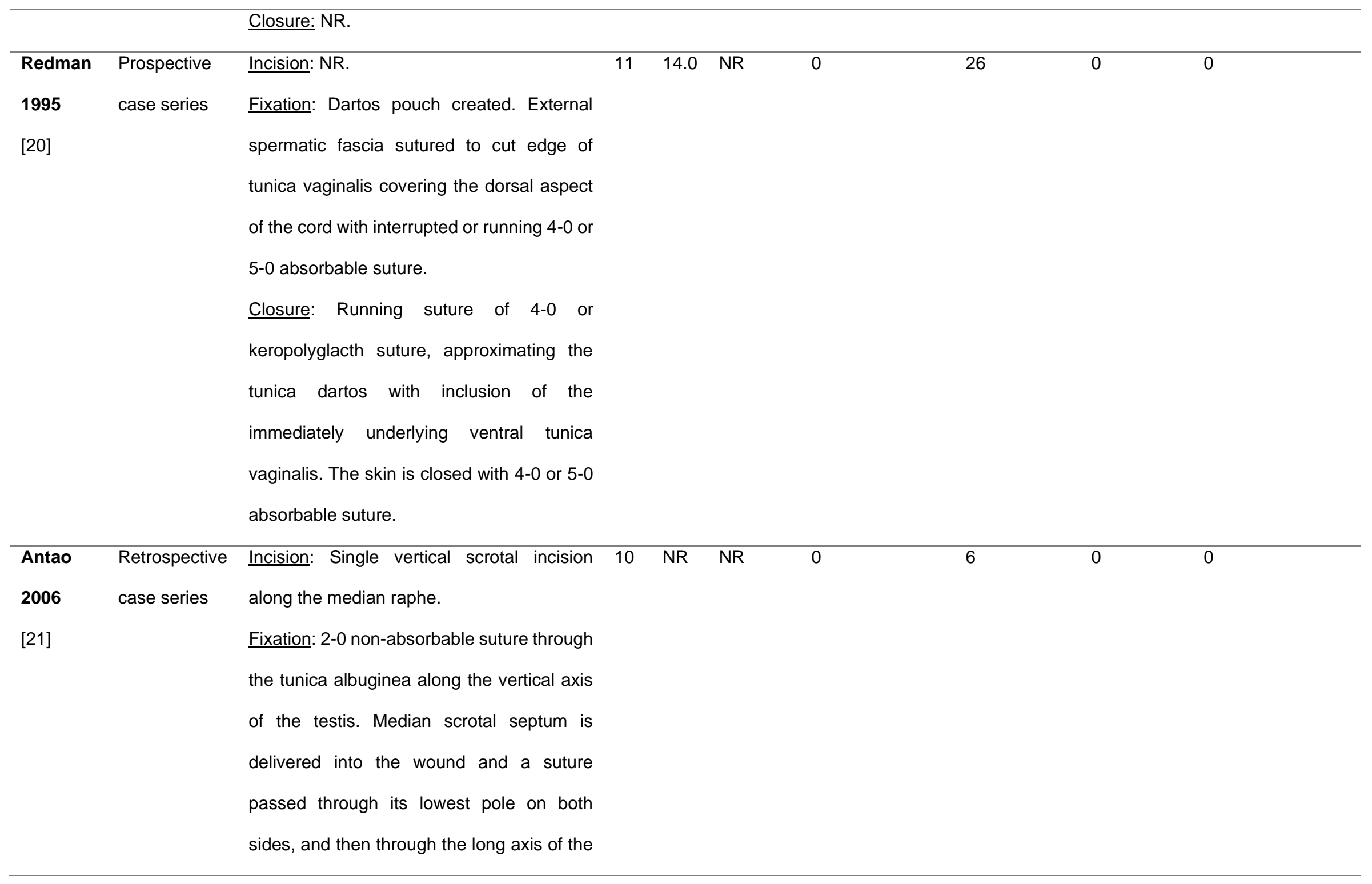


other testis from lower to upper via median

septum.

Closure: NR.

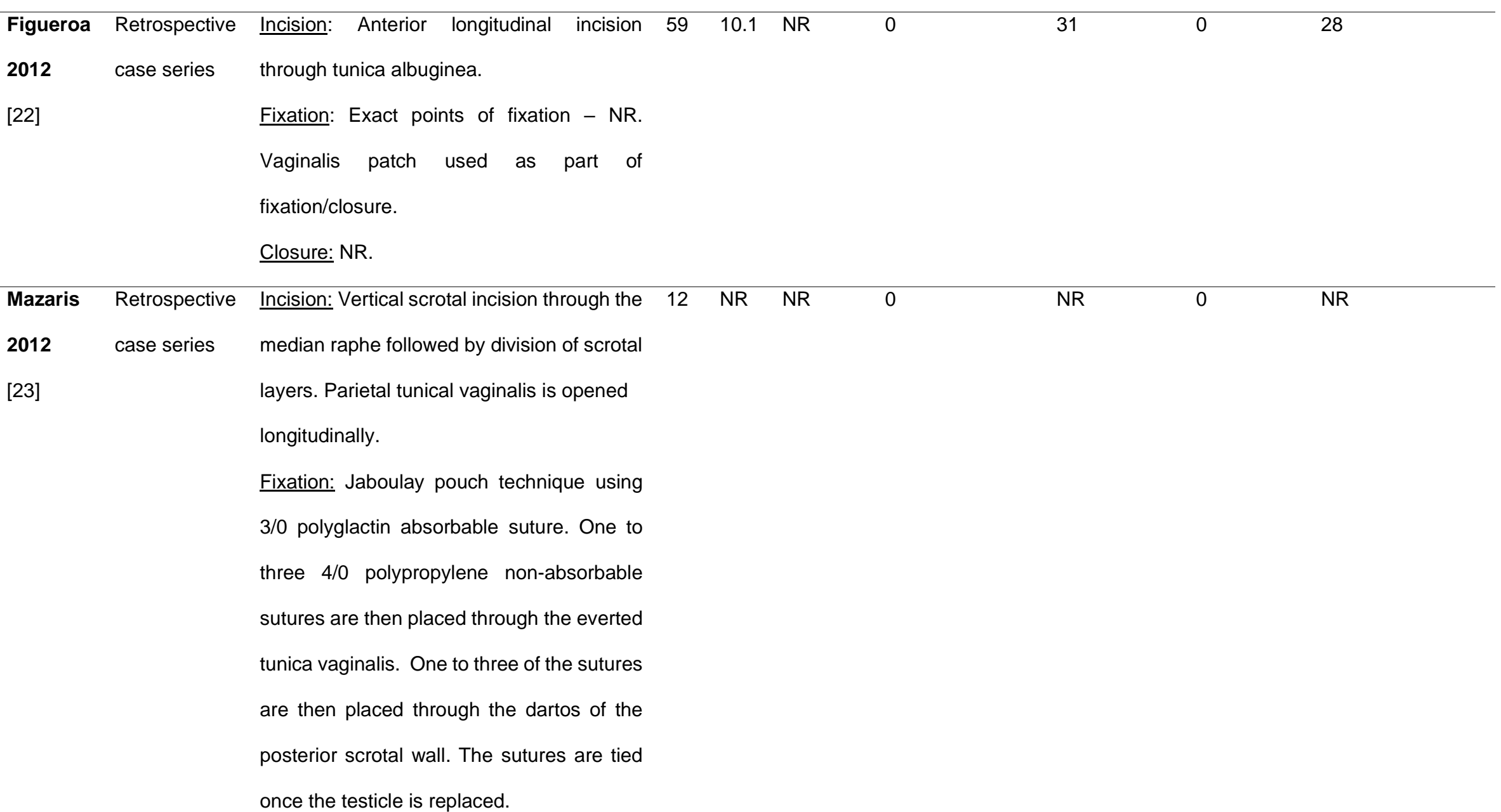


Closure: $3 / 0$ polyglactin sutures in a

continuous fashion and the edges of scrotal

skin approximated with interrupted

absorbable sutures.

Table 1 - Summary of included studies and outcomes. NR = not reported or unable to accurately discern on the basis of the information provided in the text. ${ }^{\text {in }}$ this study, thirty-five patients were included however three patients underwent orchidopexy for an indication other than acute testicular torsion. 


\section{Outcome data}

The mean duration of torsion was only reported by Figueroa et al; this was 13.4 hours [22].

Three studies documented clinic follow-up that could be attributed to the included cohort; this ranged from 6-31 weeks post-surgery [20-22]. The paper by Lent et al reported follow-up for 27 patients with a mean follow-up of six-and-a-half years, however it cannot be discerned whether this includes all patients (32/35) who had undergone treatment for acute testicular torsion rather than another indication [19]. None of the included studies reported any episodes of ipsilateral retorsion or contralateral torsion, regardless of surgical approach. Ipsilateral testicular atrophy at follow-up was noted in two studies; Greaney et al reported this in 2/22 (9.1\%) patients, while Figueroa et al reported this in $28 / 65$ (47.5\%) patients. Figueroa et al was the only study that defined testicular atrophy; this was described as $\geq 50 \%$ volume loss. $57.7 \%$ of patients underwent contralateral fixation by various methods; none of the included studies reported contralateral torsion. Fertility outcomes were not recorded in $6 / 7$ of the included studies. Though Lent et al did report fertility outcomes, the series included some patients who underwent orchidopexy for reasons other than acute testicular torsion, so it was not possible to relate outcomes to those who did have acute testicular torsion. Immediate postoperative complications were discussed in one study [17]; this reported three patients with localised inflammation, two patients who developed scrotal abscesses, and one patient who developed a stitch abscess.

\section{Discussion}

Which surgical method or suture material has the lowest rate of re-torsion?

This review highlighted the wide variation in surgical approaches for orchidopexy. The key techniques reported are described with diagrams in figures 4-10. Our principle findings were that in the 182 patients in this review, regardless of surgical approach, there was no ipsilateral or contralateral re-torsion with follow up ranging from 6-31 weeks, with follow-up up to 15 years 
in the paper by Lent et al [19]. This would suggest that, in the short term at least, all techniques included were effective and reasonable options for reducing the risk of re-torsion. A number of case reports exist in the literature regarding retorsion following orchidopexy in both animal and human models but as these are usually reported in low numbers, with a large number of potential confounders, it is difficult to draw firm conclusions on the association of one particular factor with likelihood of retorsion [24-27]. Absorbable sutures have been reported to have a higher rate of failure, although Sells et al report this may be linked to the higher number of orchidopexies carried out using absorbable sutures [24]. None of the studies included in our review reported any ipsilateral torsion and, as such, appear equal in this regard. Of note, Sells et al hypothesised on basis of animal studies [23,28-30] that the most important factor in adhesion formation is the direct apposition of tunica albuginea to the scrotal wall, with greater adhesions seen with tunica vaginalis eversion than with suture fixation of the testis alone. Lent et al confirmed that tunica vaginalis eversion and suturing alone was a valid option, though authors have suggested the anatomy conducive to torsion is not always corrected by eversion alone, so the addition of sutures might be preferable [19].

The use of testicular capsulotomy for the management of testicular compartment syndrome is not specifically reviewed in this study, although Figueroa et al do incorporate this method. Testicular capsulotomy has been demonstrated by Kutikov et al to restore normal compartment pressures in testicular compartment syndrome [31].

What are the atrophy rates of the ipsilateral testicle after fixation?

Of the three studies reporting testicular atrophy, high rates of atrophy were reported in two studies with $9 \%$ and $43 \%$ experiencing atrophy of the ipsilateral testis. The high atrophy rate $(43 \%)$ in Figueroa et al may be related to their decision to keep some testes despite minimal improvement of vascularity after detorsion. This is likely to be common practice amongst the urological community and testes that might typically be removed are given an additional 
chance to be salvaged if there was improvement in the blood flow to the affected testis after a longitudinal incision of the tunica albuginea. This finding does however suggest that testicular atrophy may be an under reported outcome following orchidopexy and should be considered more pertinently when counselling patients for scrotal exploration.

Should the contralateral testicle always be fixed?

Debate exists regarding contralateral fixation with Arnbjornsson et al arguing the low risk of contralateral torsion outweighs the risk of complications from orchidopexy [32]. In our series, $63.3 \%$ of patients underwent contralateral fixation by various methods; none of the included studies reported any contralateral torsion, but only limited medium- and long-term outcomes data were reported in all included studies. The high number of contralateral orchidopexies is likely a reflection on both case reports of contralateral torsion in cases where the contralateral testis was not fixed $[25,32-34]$. While the exact risk of contralateral torsion remains unknown, there is evidence to suggest this risk; a series of 27 cases of testicular torsion in pubertal boys found that the contralateral testis was affected by the bell clapper deformity in $78 \%$ of cases [1]. Based on this, contralateral orchidopexy would seem like a reasonable approach to take to prevent future contralateral torsion.

What is the impact of surgical technique on fertility after an episode of acute torsion?

None of the included studies where specific technique was included, reported on the fertility rates after orchidopexy for acute testicular torsion thus it is difficult to draw conclusions on whether there are any differences between different techniques. However, one study on eversion orchidopexy in 35 patients did report this, though it included 3 patients who underwent orchidopexy electively for prevention of torsion of the contralateral side and it was not possible to discern whether these fertility outcomes applied to only those who had 
orchidopexy for acute testicular torsion. Nonetheless, it is worthwhile reflecting on this study. Twenty-seven patients were followed patients for an average of 6.5 years and had paternity rates of $78 \%(7 / 9)$ in men wanting to father children. Additionally, the effect of surgical approach on testicular atrophy (reported in up to $43.1 \%$ of patients in our study) in relation to subsequent fertility was not addressed in the included studies. A study by Arap et al found no significant difference in sperm count or motility in patients who had previous orchidopexy for testicular torsion versus healthy controls [35]. In addition, a case series by Gielchinsky et al identified pregnancy rates over $90 \%$ in patients undergoing bilateral orchidopexy or orchidectomy and contralateral orchidopexy for testicular torsion. As the exact surgical techniques were not defined within these studies it is difficult to relate the findings to one specific method of orchidopexy. Despite this, some studies have discussed the possibility of suturing through the testis as a significant risk factor for spermatogenesis disorders while others have highlighted that ischaemic/reperfusion injury may compromise hormonal testicular function $[15,36]$.

\section{Summary}

There is a significant degree of heterogeneity in design, reporting and outcomes, and thus uncertainty as to optimal surgical technique, the uncertainty in long-term outcomes following orchidopexy and should be considered when counselling patients for scrotal exploration. Previously Pearce et al (2002) suggested a protocol for management of acute testicular torsion following a survey of clinical practice; recommendations included bilateral orchidopexy, the use of three non-absorbable sutures, removal of testicular appendages, and no role for additional Jaboulay repair [3]. Although this appears to be a reasonable protocol there is little evidence to back its clinical efficacy. It is likely that information such as long-term effects of technique on testicular size and fertility prognosis will be of paramount importance to the young men whom this condition typically effects. As such, there is a clearly a pressing need 
to address the question of which surgical technique, if any, causes least harm and best preserves adequate testicular function. Given this is one of the most important emergencies that Urologists deal with, the lack of high-quality data supporting specific surgical practice warrants addressing with consensus meetings amongst experts in the field and if feasible, well-designed registries and studies.

\section{Strengths and limitations of our study}

To our knowledge, this is the first systematic review assessing surgical technique for orchidopexy in testicular torsion and associated outcomes. Our review, carried out robustly according to PRISMA guidelines, highlights the absence of quality data in this area and thus the lack of evidence for the use of any particular surgical technique over another. It demonstrates a clear need for formal consensus on this topic, and the need for future studies to address these deficiencies. However, there are some limitations of the review, primarily the large degree of heterogeneity, the high risk of bias and poor reporting of outcomes in the included studies.

\section{Conclusion}

Our review demonstrates that there is currently very limited evidence in favour of any one surgical technique for acute testicular torsion, with a lack of long-term outcome data on all approaches. To adequately counsel and consent patients regarding the implications of surgery for testicular torsion, the uncertainties should be discussed. There is a need for an interim consensus until a randomised-controlled trial examining the effects of operative approach on clinical and fertility outcomes is available. 


\section{Patient summary}

Twisting of blood supply to the testis, termed testicular torsion, is a urological emergency. Testicular torsion is treated using an operation to untwist the cord that contains the blood vessels. If the testis is still salvageable, surgery can be performed to prevent further torsion. The method that is used to prevent further torsion varies. We reviewed the literature to assess outcomes of using various techniques to fix the testis. Our review shows that there is limited evidence in favour of any one technique over another.

\section{References}

1. Fehér ÁM, Bajory Z. A review of main controversial aspects of acute testicular torsion. J Acute Dis. 2016;5(1):1-8.

2. Dajusta DG, Granberg CF, Villanueva C, Baker LA. Contemporary review of testicular torsion: New concepts, emerging technologies and potential therapeutics. J Pediatr Urol. 2013;9:723-30.

3. Pearce, I; Islam, S; Mclntyre IGOK. Suspected testicular torsion: a survey of clinical practice in North West England. J R Soc Med. 2002;95:247-9.

4. Mor Y, Pinthus JH, Nadu A, Raviv G, Golomb J, Winkler H, et al. Testicular Fixation Following Torsion of the Spermatic Cord—Does it Guarantee Prevention of Recurrent Torsion Events? J Urol. 2006;175(1):171-3.

5. Zhao LC, Lautz TB, Meeks JJ, Maizels M. Pediatric Testicular Torsion Epidemiology Using a National Database: Incidence, Risk of Orchiectomy and Possible Measures Toward Improving the Quality of Care. J Urol. 2011;186(5):2009-13.

6. Leibovitch I, Mor Y. The Vicious Cycling: Bicycling Related Urogenital Disorders. Eur Urol. 2005;47(3):277-87.

7. Shteynshlyuger A, Yu J. Familial testicular torsion: A meta analysis suggests 
inheritance. J Pediatr Urol. 2013;9(5):683-90.

8. Seng YJ, Moissinac K. Trauma induced testicular torsion: a reminder for the unwary. J Accid Emerg Med. 2000;17(5):381-2.

9. Kapoor S. Testicular torsion: a race against time. Int J Clin Pract. 2008;62(5):821-7.

10. Somani BK, Watson G, Townell N. Testicular torsion. BMJ. 2010;341:c3213.

11. Mansbach JM, Forbes P, Peters C. Testicular torsion and risk factors for orchiectomy. Arch Pediatr Adolesc Med. 2005;159(12):1167.

12. Cost NG, Bush NC, Barber TD, Huang R, Baker LA. Pediatric Testicular Torsion: Demographics of National Orchiopexy Versus Orchiectomy Rates. J Urol. 2011;185(6):2459-63.

13. Martin AD, Rushton HG. The Prevalence of Bell Clapper Anomaly in the Solitary Testis in Cases of Prior Perinatal Torsion. J Urol. 2014;191(5):1573-7.

14. Bolln C, Driver CP, Youngson GG. Operative management of testicular torsion: Current practice within the UK and Ireland. J Pediatr Urol. 2006;2(3):190-3.

15. Coughlin MT, Bellinger MF, LaPorte RE, Lee PA. Testicular suture: a significant risk factor for infertility among formerly cryptorchid men. J Pediatr Surg. 1998;33(12):1790-3.

16. Osman NI, Collins GN. Urological litigation in the UK National Health Service (NHS): an analysis of 14 years of successful claims. BJU Int. 2011;108(2):162-5.

17. Greaney G. Torsion of the testis : a review of 22 cases. Br J Surg. 1975;62:57-8.

18. Douglas LL. Simple technique for testicular fixation in management of torsion. Urology. 1988;XXXII(4):352-3.

19. Lent V, Stephani A. Eversion of the Tunica Vaginalis for Prophylaxis of Testicular 
Torsion Recurrences. J Urol. 1993;150:1419-1421.

20. Redman JF, Barthold JS. A technique for atraumatic scrotal pouch orchiopexy in the management of testicular torsion. J Urol. 1995;154:1511-2.

21. Antao, B; MacKinnon AE. Axial fixation of testes for prevention of recurrent testicular torsion. Surgeon. 2006;4(1):20-1.

22. Figueroa V, Salle JLP, Braga LHP, Romao R, Koyle MA, Bägli DJ, et al. Comparative Analysis of Detorsion Alone Versus Detorsion and Tunica Albuginea Decompression ( Fasciotomy ) with Tunica Vaginalis Flap Coverage in the Surgical Management of Prolonged Testicular Ischemia. J Urol. 2012;188(4):1417-23.

23. Mazaris E, Tadtayev S, Shah T, Boustead G. Surgery illustrated Focus on details a novel method of scrotal orchidopexy: Description of the technique and short-term outcomes. BJU Int. 2012;110(11):1838-42.

24. Sells H, Moretti KL, Burfield GD. Recurrent torsion after previous testicular fixation. Vol. 72, ANZ Journal of Surgery. John Wiley \& Sons, Ltd; 2002:46-8.

25. C. von Zastrow, J.A. Sotelino Recurrent testicular torsion: is retorsion of a fixed testis possible? A case report and literature review. Urologe A. 2005;44(11):1337-1340.

26. Koochakzadeh S, Johnson K, Rich MA, Swana HS. Testicular torsion after previous surgical fixation. J Pediatr Surg Case Reports. 2019 1;47:101225.

27. K. Chondros, N. Chondros Recurrent testicular torsion after orchidopexy Pan Afr Med J, 20 (2015), p. 190

28. Gandhi J, Dagur G, Sheynkin YR, Smith NL, Khan SA. Testicular compartment syndrome: An overview of pathophysiology, etiology, evaluation, and management. Vol. 5, Translational Andrology and Urology. AME Publishing Company; 2016:92734. 
29. Morse TS, Hollabaugh RS. The 'window' orchidopexy for prevention of testicular torsion. J Pediatr Surg 1977;12: 237-40.

30. Rodrigues LE, Kaplan GW. An Experimental Study of Methods to Produce Intrascrotal Testicular Fixation. J Urol 1988;139(3):565-7.

31. Kutikov A, Casale P, White MA, Meyer WA, Chang A, Gosalbez R, et al. Testicular Compartment Syndrome: A New Approach to Conceptualizing and Managing Testicular Torsion. Urology. 2008;72(4):786-9.

32. Arnbjörnsson E, Kullendorff CM. Testicular torsion in children--bilateral or unilateral operation. Acta Chir Scand. 1985;151(5):425-7.

33. Rasmussen JS. Contralateral testicular torsion after previous unilateral orchiopexy for undescended testis. Scand J Urol Nephrol. 1996;30(6):513-4.

34. Ransler CW, Allen TD. Torsion of the spermatic cord. Urol Clin North Am. $1982 ; 9(2): 245-50$.

35. Arap MA, Vicentini FC, Cocuzza M, Hallak J, Athayde K, Lucon AM, et al. Late Hormonal Levels, Semen Parameters, and Presence of Antisperm Antibodies in Patients Treated for Testicular Torsion. J Androl. 2007;28(4):528-32.

36. Romeo C, Impellizzeri P, Arrigo T, Antonuccio P, Valenzise M, Mirabelli S, et al. Late hormonal function after testicular torsion. J Pediatr Surg. 2010;45(2):411-3. 